\title{
Electromagnetic Wave Scattering From a Cylinder Immersed in a Warm Plasma ${ }^{1}$
}

\author{
Edmund K. Miller \\ High Altitude Engineering Laboratory, Department of Aerospace Engineering, \\ University of Michigan, Ann Arbor, Mich. 48105, U.S.A.
}

(Received March 31, 1967; revised May 4, 1967)

\begin{abstract}
The scattering of transverse electric (TE; $E_{z}=0$ ) and transverse magnetic (TM; $H_{z}=0$ ) plane waves from a perfectly conducting cylinder located in a warm (compressible) plasma is considered. Numerical values for the total primary cross sections (TE-TE and TM-TM scattering) are given. Results for the ratios of the total conversion cross sections (TE-TM, TM-TE, TE-electrokinetic (EK) and $T \bar{M}-E K$ scattering) to the corresponding primary cross section are also presented. The effect of varying the cylinder radius, plasma frequency, electron temperature, and angle of incidence are shown. A comparison is also made of results obtained from a vacuum-sheath model with those due to a more realistic inhomogeneous sheath model. It is found that, except for near-grazing angles of incidence, for cylinder radii much less than the EM wavelength or the plasma frequency nearly equal to the incident wave frequency, the conversion cross sections are on the order of $10^{-2}$ or less of the primary cross sections. The primary cross sections are, at the same time, not appreciably affected by the sheath or nonzero temperature.
\end{abstract}

\section{Introduction}

There is currently a great deal of interest, in connection with radiation and scattering problems involving plasmas, to take into account the nonzero temperature of the plasma. This is because the warm plasma is compressible and can support, in addition to the electromagnetic (EM) wave, an electron acoustic wave. The coupling of this electron pressure wave, or as it will be referred to here the electrokinetic (EK) wave, to the EM wave, may give rise to effects which cannot be explained by the usual cold-plasma theory, where the plasma is characterized by an equivalent permittivity. One example of this is the scattering cross section of a plasma cỳlinder, which exhibits resonances that can be accounted for only if the temperature of the plasma is taken into account.

It is natural to ask whether the nonzero plasma temperature will similarly have a perturbing effect on the scattering cross section of an obstacle immersed in a plasma. For example, it may be shown (Wait, 1965) that the transverse electric (TE) polarization of the EM wave, upon scattering from a perfectly conducting, infinite cylinder in free space, produces only a scattered TE wave. A similar result holds for the incident transverse magnetic (TM) wave, in that only a scattered TM wave will be produced. The temperature of the plasma can alter this situation, however, leading to conversion of TE to TM waves and TM to TE waves, as well as to scattered EK waves.

There is an additional mechanism which may lead to this polarization conversion, or cross coupling as we will refer to it here, between the incident wave and

\footnotetext{
Vork in this paper was sponsored by NASA under contract No. NASr-54(05).
}

the scattered fields. That is the sheath which forms about a body in a plasma. (This sheath is of course a manifestation of the nonzero plasma temperature.) If the body is allowed to reach its floating potential in the plasma, as will be assumed here, the sheath is a region of electron deficiency, which may extend sev. eral electron Debye lengths into the plasma from the body. This is why, in dealing with EM waves in compressible plassmas, in many treatments the sheath has been approximated by a free-space layer (the vacuum sheath) between the uniform plasma and the body. A more accurate representation of the sheath. of course. would be one where the nonuniformity of the plasma within it is taken into account.

It is the purpose of this paper to investigate the effect of the plasma compressibility and sheath upon the scattering cross section of an infinite, perfectly conducting cylinder, immersed in a plasma. The sheath will be represented in two ways, using the vacuum sheath model mentioned above, and a more realistic representation which takes the sheath inhomogeneity into account, the inhomogeneous sheath model. In both models, the sheath will be assumed to be of finite thickness, with the plasma external to the sheath uniform throughout. The theoretical development will be treated rather briefly, since the details have been presented elsewhere, in favor of giving the more interesting aspects of the numerical results in greater detail. A theoretical discussion in greater detail is given by Miller (1966) and Miller and Olte (1966).

\section{Formulation}

The linearized hydrodynamic equations for the electrons (the ion motion is neglected), together with 
Maxwell's equations, serve to describe the time-varying, or dynamic, field behavior in the plasma. It is assumed that there are no externally applied fields, and that collisional, viscous and gravitational force effects may be neglected. In the case of the vacuum sheath, modal expressions for each of the incident and scattered fields, both in the uniform plasma and vacuum sheath, are readily obtained in terms of Fourier series involving cylindrical Bessel functions and the unknown Fourier coefficients, which are to be obtained from the boundary conditions. For the inhomogeneous sheath model, solutions in the uniform plasma are similarly obtained, but the field equations for the sheath region require numerical integration. In either case, however, the scattering cross sections, the quantities of interest here, are obtained from the Fourier coefficients for the scattered fields in the uniform plasma.

The Fourier coefficients for the fields produced by the incident wave are obtained from the boundarycondition equations. The boundary conditions used for the vacuum sheath model are continuity of the tangential electric and magnetic fields at the sheathplasma interface, vanishing of the tangential electric field on the cylinder, and either vanishing of the normal dynamic electron velocity (the hard boundary) or vanishing of the dynamic electron number density (the soft boundary) at the sheath-plasma interface. The latter boundary condition could be replaced by an admittance-boundary condition relating the dynamic electron number density and velocity, where the surface admittance is arbitrary. We have chosen to investigate only the extreme values of the range of possible admittance values, for want of a meaningful way to otherwise specify the surface admittance, and recognizing that the cross-coupling is maximized by the admittedly over-simplified hardboundary condition. Our primary concern in this connection is to determine whether such cross-coupling effects are at all significant in the most favorable of conditions, so that the use of the hard-boundary condition is felt to be justified. It is worthwhile to note that, in the case of a soft boundary, there is no coupling between the EM and EK waves at the boundary, and thus no EM-EK coupling for the vacuum-sheath model. Even in the case of the soft boundary, however, there exists TE-TM and TM-TE coupling for a vacuum sheath.

The boundary conditions for the inhomogeneoussheath model are the same as those for the vacuum sheath mentioned, with the exception that the normal dynamic electron velocity and dynamic electron number density be continuous at the sheath-uniform plasma interface. In addition, the boundary condition applied at the vacuum sheath-uniform plasma interface between the ${ }^{-}$dynamic electron number density and velocity is now applied at the cylinder surface, since the plasma extends to the cylinder. In this case, the use of the soft-boundary condition no longer means that the $E M$ and $E K$ waves are uncoupled, since the sheath inhomogeneity also produces coupling between them.

The differential scattering cross-sections unit length of the cylinder are then given by

$$
\begin{gathered}
\sigma_{--}^{D}=\frac{4 \lambda_{E}^{2}}{K_{E}^{3}}\left|\sum_{n=-\infty}^{n=+\infty} e^{(-i n \varphi)} i^{n} A_{n--}^{S}\right|^{2}, \\
\sigma_{-p}^{D}=\frac{4}{N^{2} K_{E}}\left|\sum_{n=-\infty}^{n=+\infty} e^{(-i n \omega)} i^{n} A_{n-p}^{S}\right|^{2},
\end{gathered}
$$

where the first dash subscript on $\sigma$ and the Fourier coefficient for the scattered field $A^{s}$ will be an $e$ or $m$, denoting the incident wave to be either $\operatorname{TE}\left(E_{z}=0\right)$ or $\operatorname{TM}\left(H_{z}=0\right)$. The second dash subscript indicates the type of scattered wave, with $p$ denoting the EK wave. The cylinder is oriented with its axis coincident with the $z$ axis of a cylindrical $(\rho, \varphi, z)$ coordinate system, in which the azimuthal angle $\varphi$ is measured from the plane formed by the $z$ axis and the incident plane-wave propagation vector, with the backscatter direction given by $\varphi=0^{\circ}$. The other quantities are defined by

$$
\begin{aligned}
N & =\omega_{p} / \omega=f_{p} / f \\
K_{E} & =\frac{\omega}{v_{l}} \sqrt{1-N^{2}}=K_{E 0} \sqrt{1-N^{2}} \\
\lambda_{E} & =K_{E} \sqrt{1-\cos ^{2} \theta^{i}}
\end{aligned}
$$

where $f_{p}$ is the plasma frequency, $f$ the incident wave frequency, $v_{l}$ is the velocity of light in free space, and $\theta^{i}$ is the angle of incidence measured from the positive $z$ axis. Expressions for the $A_{n--}^{s}$ are given by Miller (1966), for the vacuum-sheath model. The scattering coefficients for the inhomogeneous sheath are obtained by numerical integration, which is also discussed by Miller (1966).

The total scattering cross sections are obtained by integrating (1), multiplied by $1 / 2 \pi$, over $\vartheta$ from $-\pi$ to $\pi$ with the result

$$
\begin{aligned}
& \sigma_{T}^{T}=\frac{4 \lambda_{E}^{2}}{K_{E}^{3}} \sum_{n=-\infty}^{n=x}\left|A_{n-\cdot}\right|^{2}, \\
& \sigma_{-p}^{T}=\frac{4}{N^{2} K_{E}} \sum_{n=-\infty}^{n=\infty}\left|A_{n-p}\right|^{2} .
\end{aligned}
$$

For purposes of investigating the conversion or coupling of TE to TM energy, TM to TE energy, and EM to EK energy, we define the coupling coefficients:

$$
\begin{aligned}
& C_{e m} \equiv \sigma_{e m}^{T} / \sigma_{e e}^{T} \\
& C_{m e} \equiv \sigma_{m e}^{T} / \sigma_{m m}^{T}
\end{aligned}
$$




$$
\begin{gathered}
C_{e p} \equiv \sigma_{e p}^{T} / \sigma_{e e}^{T} \\
\bar{C}_{m p} \equiv \sigma_{m p}^{T} / \sigma_{m m}^{T} .
\end{gathered}
$$

The coupling coefficients thus represent the amount of energy scattered into fields of polarization different from that of the incident wave, in comparison with the scattered energy having the polarization of the incident wave. They should be useful in indicating the perturbing effect of the sheath and plasma compressibility on the scattering cross section with the incident wave polarization. In the next section are presented numerical results for the cross sections and coupling coefficients. For convenience, we will refer to $\sigma_{e e}^{T}$ and $\sigma_{m m}^{T}$ as the primary cross sections, and will call the others conversion cross sections in the following.

\section{Numerical Results}

There are a number of parameters which are of interest in connection with the effect their variation may have on the cross sections and coupling coeff. cients. Perhaps the two most significant quantities which affect the coupling coefficients are the sheath thickness and electron temperature, since when both of these are zero, the coupling coefficients are also zero. Consequently, we present in figures 1 and 2, respectively, the variation of the four coupling coefficients with sheath thickness $X$ and the electron temperature $T$ in ${ }^{\circ} \mathrm{K}$. The sheath thickness $X$ is in: units of the electron Debye length $\left(D_{l}\right)$. The incident wave frequency in $1 \mathrm{GHz}, N=0.7, \theta^{i}=\pi / 4$, and the cylinder radius $c$ is $5 \mathrm{~cm}$.

There are curves plotted on figure 1 for results obtained from both the vacuum-sheath model and the inhomogeneous-sheath model for both the hard and soft boundaries. In the case of the inhomogeneous sheath, the static potential is taken to be of parabolic form, having the value $-5.34 \mathrm{~V}$ at the cylinder, corresponding to a mercury plasma with $T=10^{4}{ }^{\circ} \mathrm{K}$ (see Miller and Olte, 1966). The parabolic form for the potential is chosen since some experimental measure. ments by Gabor, Ash, and Dracott (1955), Gierke, Ott, and Schwirzke (1961), and Harp and Kino (1963), show the static sheath potential to be approximately parabolic. It should be noted that the static potential becomes zero and the plasma consequently is uniform at the radius $\rho=c+X D_{\ell}$ for the inhomogeneoussheath model, and that the sheath thickness for both the vacuum and inhomogeneous sheaths is $X D_{\ell}$ Two curves are shown for each of the EM-EM coupling coefficients from the vacuum-sheath model, one for the hard boundary and the other for the soft boundary, while the EM-EK coupling coefficients are shown, of course, for the hard boundary only. If we first observe the EM-EM coupling coefficients, we see that there is a rapid increase in the coupling coefficient with increasing vacuum-sheath thickness, the increase being approximately $(X / 2)^{2}$ referred to the $X=0$ case, for the hard boundary. For the soft boundary (equivalent to a zero-temperature plasma), the coupling coefficients are substantially the same as those for the hard boundary, for $X>5$, but for thinner sheaths there is a progressively increasing difference between the results for the two boundary conditions as $X$ tends toward zero.

The inhomogeneous sheath EM-EM coupling coefficients are seen to increase with increasing sheath thickness in a way similar to the vacuum-sheath results, for $X>2$, and the hard boundary, with a vacuumsheath thickness approximately 0.6 the inhomogeneous-sheath thickness leading to the same value for the coupling coefficient. In addition, the difference between the hard and soft boundary results for the inhomogeneous sheath is similar to that observed for the vacuum sheath for $X>5$. For sheath thicknesses less than about 4 , however, all the coupling coefficients exhibited an oscillatory behavior with decreasing $X$,

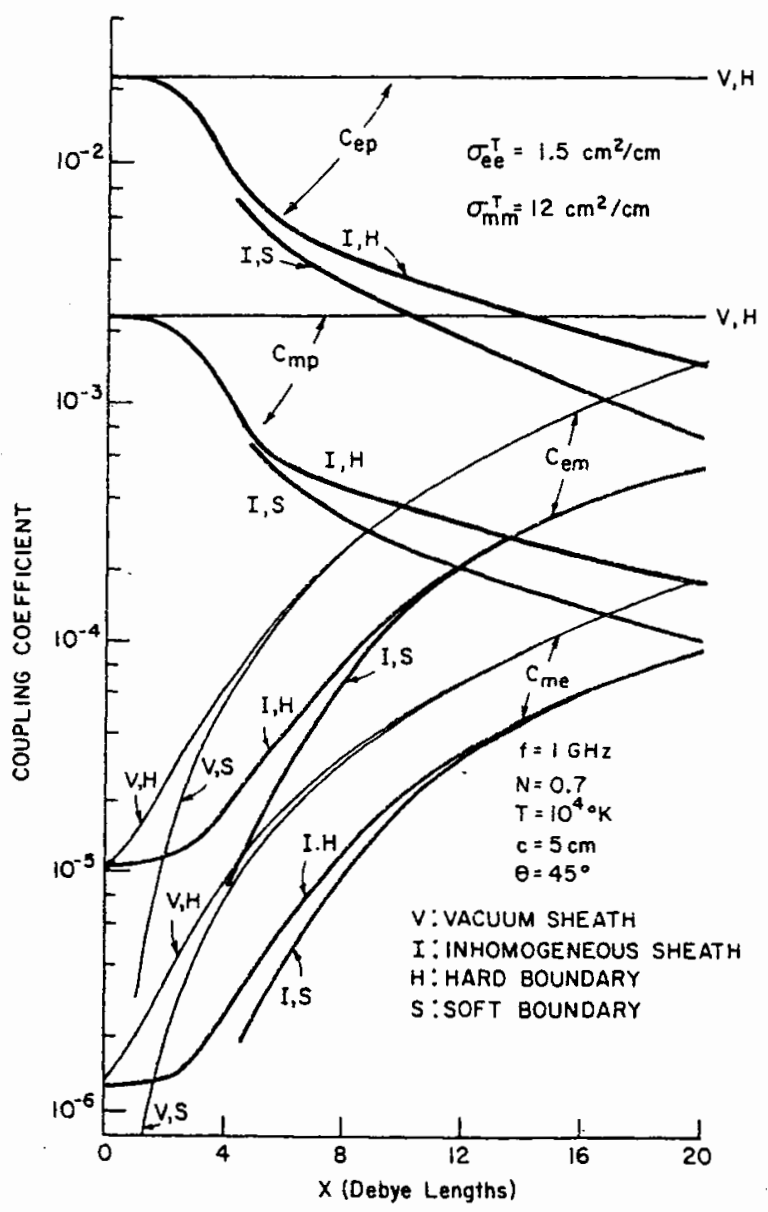

FIGURE 1. The coupling coefficients as a function of sheath thickness X for both the vacuum-sheath and inhomogeneoussheath models and the soft and hard boundaries. 
for the soft boundary, sometimes becoming larger than the hard-boundary results. Since the calculations required to accurately obtain this area of the curve would have been quite time-consuming, this portion of the curves is not plotted. The reason for this oscillatory behavior of the soft-boundary results is not clear but may be due to resonance effects in the EK wave, which is excited. This is a point that may be worth investigating further.

When we turn our attention to the EM-EK coupling coefficients, we find that the vacuum-sheath values are unaffected by changing sheath thickness, while the inhomogeneous-sheath results show a generally decreasing coupling with increasing sheath thickness. As a result, near $X=20$, the EM-EK coupling coefficients for the inhomogeneous sheath are the same order of magnitude as the EM-EM coupling coeffcients, while exceeding the EM-EM coupling coefficients by several orders to magnitude near $X=0$. Contrary to the case of the EM-EM coupling coefficients, for $X>5$, the hard-boundary and soft-boundary results for the EM-EK coupling coefficients and the inhomogeneous sheath diverge in value with increasing $X$.

It is of interest to note that neither $\sigma_{e e}^{\tau_{e}}$ nor $\sigma_{\mathrm{mm}}$ vary significantly from their sheathless value with changing $X$ for both the vacuum-sheath and inhomogeneoussheath models, the change being less than 1 percent for $X$ increasing from 0 to 20 . Their sheathless values are $\sigma_{e e}^{T}=1.5 \mathrm{~cm}^{2} / \mathrm{cm}$ and $\sigma_{m m}^{T}=12 \mathrm{~cm}^{2} / \mathrm{cm}$, so that the conversion cross sections can be obtained from figure 1 using $(5)-(8)$.

Since the soft boundary results vary only slightly from the hard boundary results for $X>4$ on figure 1 , subsequent results are shown for the hard boundary only. In addition, since the inhomogeneous-sheath and vacuum-sheath results are similar for the EM-EM coupling coefficients, the graphs to follow will be for the vacuum-sheath model only. We note that the EM-EK coupling coefficients thus obtained from the vacuum-sheath model will likely be somewhat exaggerated compared with values that would be obtained from the inhomogeneous sheath.

In figure 2, where the electron temperature is the independent variable, results are given for the vacuumsheath model and the hard boundary only, for $X=0$ and 10. Note the absolute sheath thickness is now changing in proportion to the square root of the temperature, since $D_{\ell}=v_{r} /\left(\sqrt{3 \omega_{p}}\right)$ where $v_{r}$ is the rms electron velocity. Since there is no difference between the $X=0$ and $X=10$ case for $C_{e p}$ and $C_{m p}$, only one curve is shown for each of these two quantities. It may be observed that the EM-EM coupling coefficients vary in proportion to the electron temperature $T$, while the EM-EK coupling coefficients vary as the square root of $T$. Again $\sigma_{e e}^{T}$ and $\sigma_{m m}^{T}$ were found to be nearly constant at their sheathless values, over the range of $T$ shown, having the values given above.

The coupling coefficients are plotted as a function of $\theta^{i}$, the angle of incidence, in figure 3 , for the vacuum-

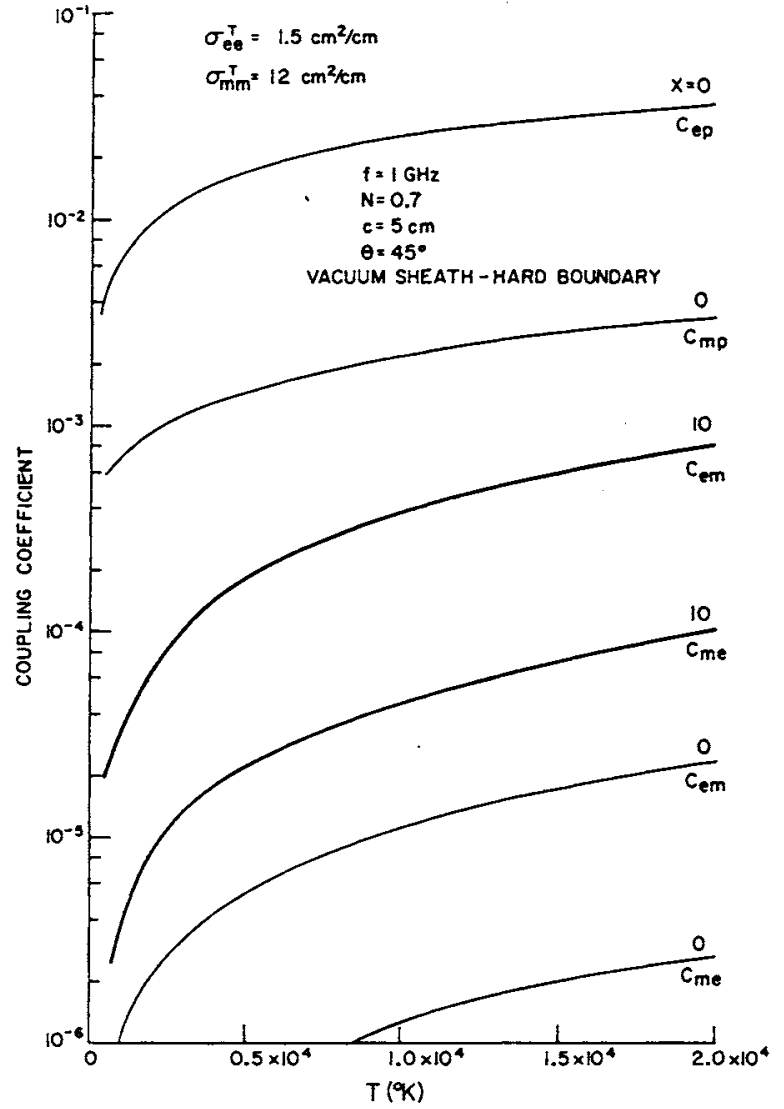

FIGURE 2. The coupling coefficients as a function of electron temperature $\mathrm{T}$ for the vacuum-sheath model and the hard boundary.

sheath model and hard boundary, again for $X=0$ and. 10 , with $T=10^{4} \mathrm{~K}$ and the other parameter values as for figure 1. We may observe that the coupling coefficients exhibit a decreasing trend as $\theta^{i}$ tends to normal incidence, and with the exception of $C_{e p}$, become zero at $\theta^{i}=\pi / 2$. We may also see that the effect of the sheath is lessened for $C_{e m}$, and remains unchanged for $C_{m e}$, as $\theta^{i}$ tends towards zero. On the other hand, $C_{e p}$ begins to show a noticeable sheath effect near grazing incidence. Because $\sigma_{e e}^{\tau}$ and $\sigma_{m m}^{T}$ vary with $\theta^{i}$, these cross sections are shown in figure 4 , as a function of $\theta^{i}$. It may be observed in figure 4 that the $10 D_{\ell}$ thick vacuum sheath does influence the cross section for near grazing angles of incidence, increasing $\sigma_{e e}^{T}$ and decreasing $\sigma_{m m}^{T}$ with respect to the sheathless case. (The values for $C_{e p}$ of figure 3 and $\sigma_{e e}^{T}$ of figure 4 for $\theta^{i}=90^{\circ}$ are consistent with the results of Seshadri, Morris, and Mailloux (1964), within the limits of their graphical accuracy. Since their analysis is restricted to normal incidence, this is the only case where a direct comparison can be made.) 


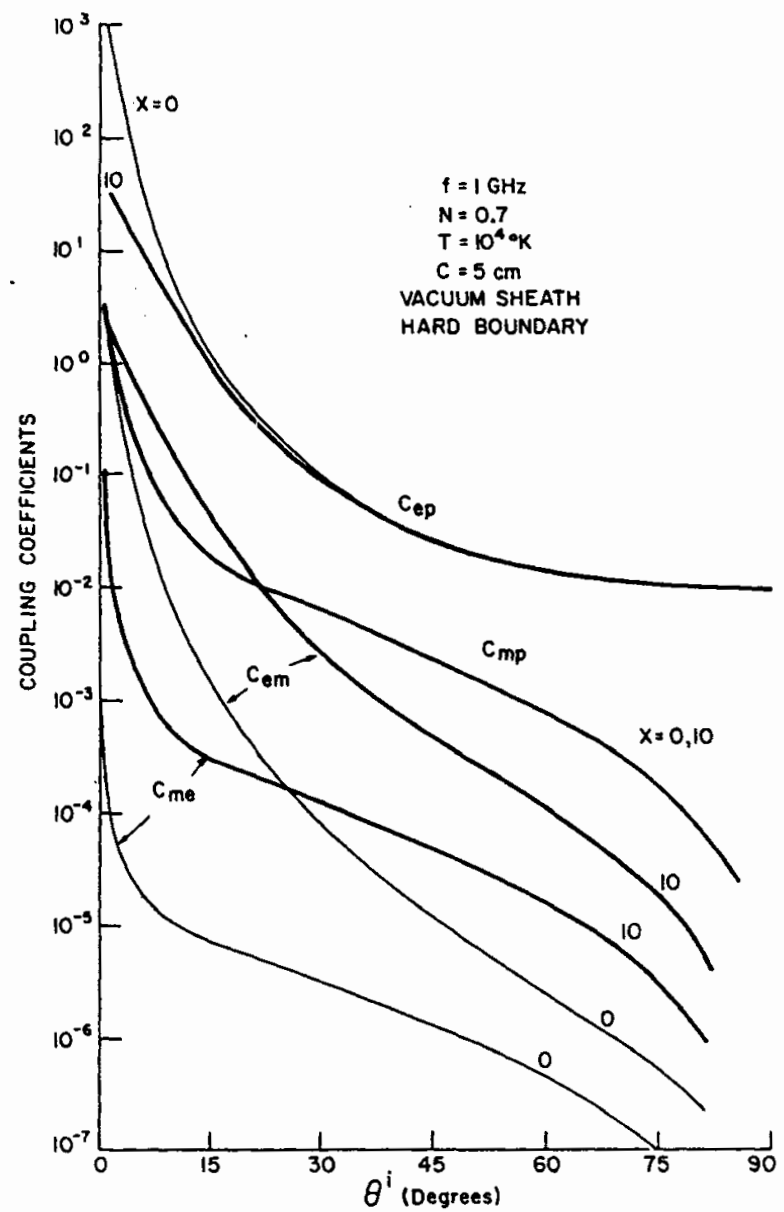

FIGURE 3. The coupling coefficients as a function of angle of incidence $\theta^{i}$ measured from the cylinder axis for the vacuum-sheath model and the hard boundary.

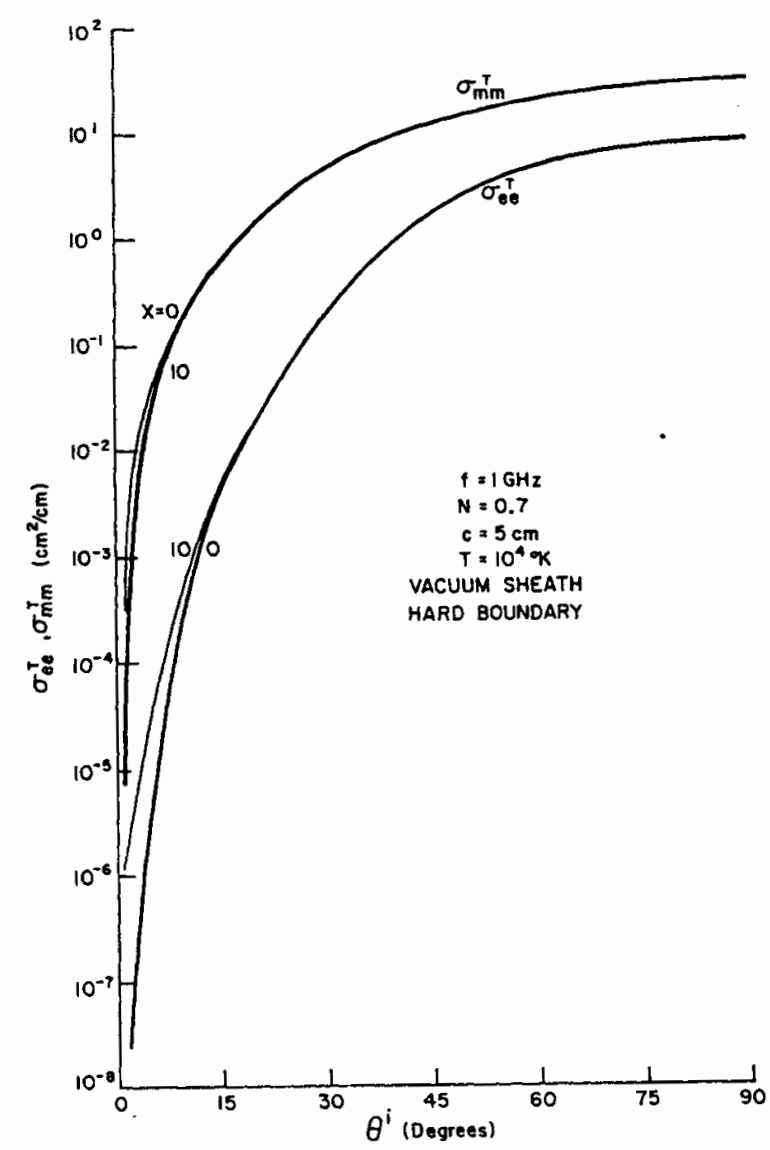

FigURE 4. The primary cross sections as a function of angle of incidence $\theta^{i}$ for the vacuum sheath model and the hard boundary.
The cylinder radius $c$ is the independent variable in figures 5 and 6 , in which are shown, respectively, the coupling coefficients and the primary cross sections $\sigma_{e e}^{T}$ and $\sigma_{m m}^{T}$ for the vacuum-sheath model, and hard boundary for $X=0$ and $X=10$, with the other parameter values the same as for figure 1 . The curves of figures 5 and 6 are drawn as dashed lines since the calculated points were not obtained close enough together to show the fine structure of the curves, but only to show the trend of the data with increasing cylinder radius $c$.

We see in figure 5 that the coupling coefficients show generally decreasing values with increasing cylinder radius. Since the TE wave decouples from the TM and EK waves for scattering from an infinite plane, we should expect that all the coupling coefficients except $C_{m p}$ would become zero, if the radius $c$ were given larger and larger values. The large increase in $C_{e p}$, $C_{e m}$, and $C_{m p}$, as $c$ approaches zero, reflects the fact that the corresponding conversion cross sections, while tending toward zero, do so more slowly than the primary cross sections. It can be shown, for ex. ample, that as $c \rightarrow 0, C_{e \nu} \rightarrow\left(v_{t} / v_{r}\right)^{2} / N^{2}$, from a small argument expansion of the scattering coefficients. This compares with $C_{e p} \rightarrow\left(v_{t} / v_{r}\right)^{3} / N^{2}$ for the scattering from a spherical plasma blob in the Rayleigh region, a result derived by Cohen (1962).

It is apparent that for large enough cylinder radii, $C_{e m}$ and $C_{m e}$ are approaching the same values, reflecting the fact that TM-TE and TE-TM coupling occurs with the same efficiency relative to the primary cross sections. As a matter of interest, $\sigma_{e m}^{r}$ and $\sigma_{m e}^{T}$ are practically equal regardless of the cylinder radius and sheath thickness, but for the smaller radii, $\sigma_{e e}^{T}$ is less than $\sigma_{m m}^{T}$ (shown in fig. 6), producing a corresponding difference in the coupling coefficients $C_{e m}$ and $C_{m e}$. We also observe in figure 5 that the effect of the sheath on the EM-EM coupling coefficients appears to be independent of the cylinder radius, for 
the range investigated. The EM-EK coupling coefficients, on the other hand, begin to exhibit a slight sheath dependence at the larger values of cylinder radii.

The final graphs of this series, figures 7 and 8 , show respectively, the coupling coefficients and the primary cross sections $\sigma_{e e}^{T}$ and $\sigma_{m m}^{T}$ as functions of $N$, the ratio of. $f_{p} / f$, with the other parameter values used for figure 1 , and the vacuum sheath thicknesses of $X=0$ and $X=10$. We note that the TM-TE coupling coefficients of figure 7 and the TE-TE primary cross section of figure 8 exhibit decreasing values with increasing $N$, while the converse behavior is true of the other coupling coefficients and the TM-TM primary cross section. In addition, the sheath effect upon the coupling coefficients is observed to decrease with increasing $N$, a result to be expected since the sheath thickness relative to the wavelength is decreasing.

\section{Summary and Conclusion}

The results of this investigation may be briefly summarized as follows:

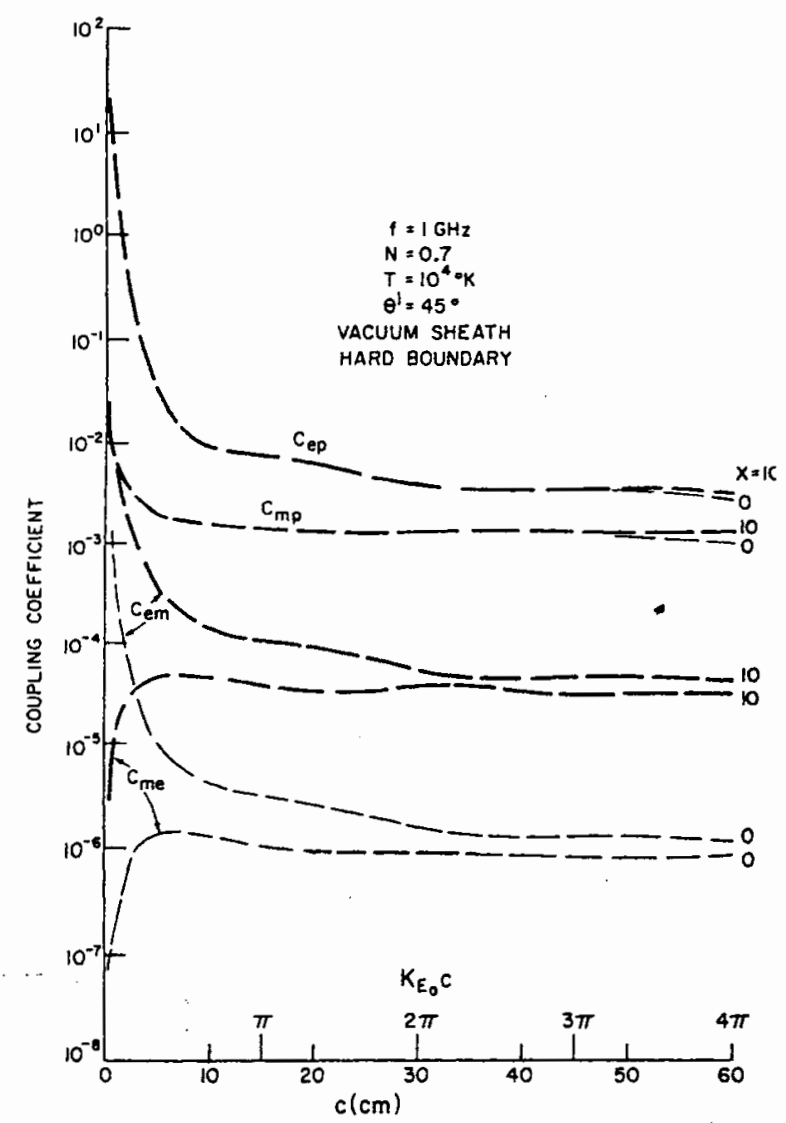

FIGURE 5. The coupling coefficients as a function of cylinder radius $\mathrm{c}$ for the vacuum-sheath model and the hard boundary. (a) The primary cross sections are negligibly affected by the plasma compressibility and sheath, except at near grazing angles of incidence $\left(\theta^{i}<15^{\circ}\right)$, for $N$ near unity or $K_{E 0} c \ll 1$, situations where the coupling coefficients approach unity.

(b) The EM-EM coupling coefficients increase approximately in proportion to the electron temperature and to the square of the vacuum-sheath thickness expressed in electron Debye lengths. The EM-EK coupling coefficients vary as roughly the square root of the electron temperature and are practically unaffected by the vacuum sheath. The coupling coefficients are found to be less affected by the vacuum sheath as $f_{p} / f$ approaches unity.

(c) With the exception of $C_{e p}$, the coupling coefficients are less than $10^{-2}$, except for near-grazing incidence, for $N$ near unity or $K_{E 0} c \ll 1$.

(d) The inhomogeneous sheath primary cross sections and EM-EM coupling coefficients are quantitatively similar to the vacuum-sheath results, with a vacuum sheath approximately 0.6 the inhomogeneoussheath thickness (for the inhomogeneous-sheath model used), producing vacuum sheath coupling coefficients

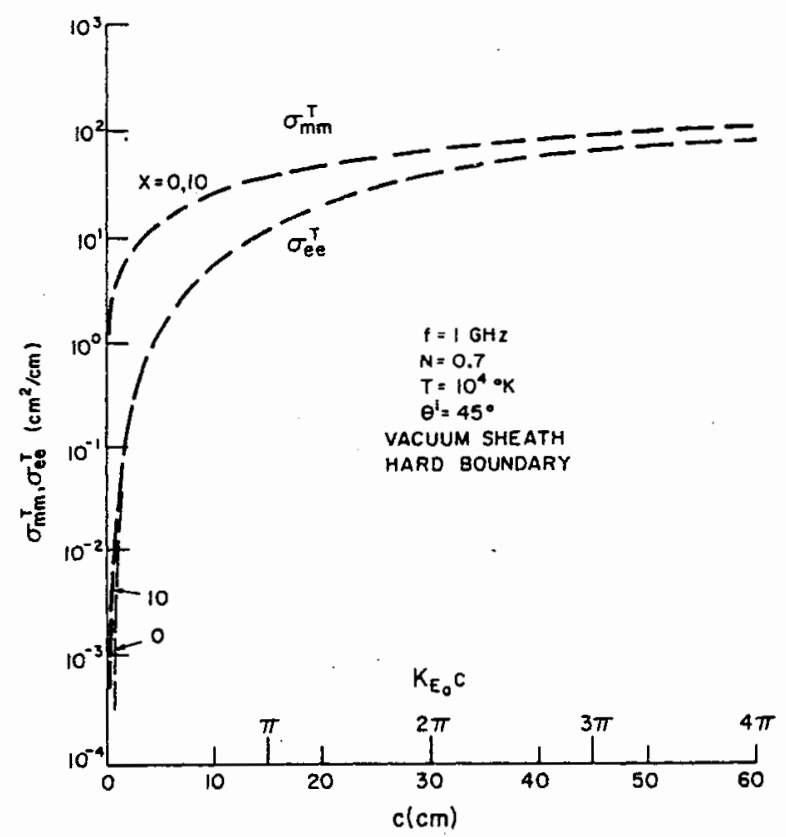

FIGURE 6. The primary cross sections as a function of cylinder radius $\mathrm{c}$ for the vacuum-sheath model and the hard boundary. 


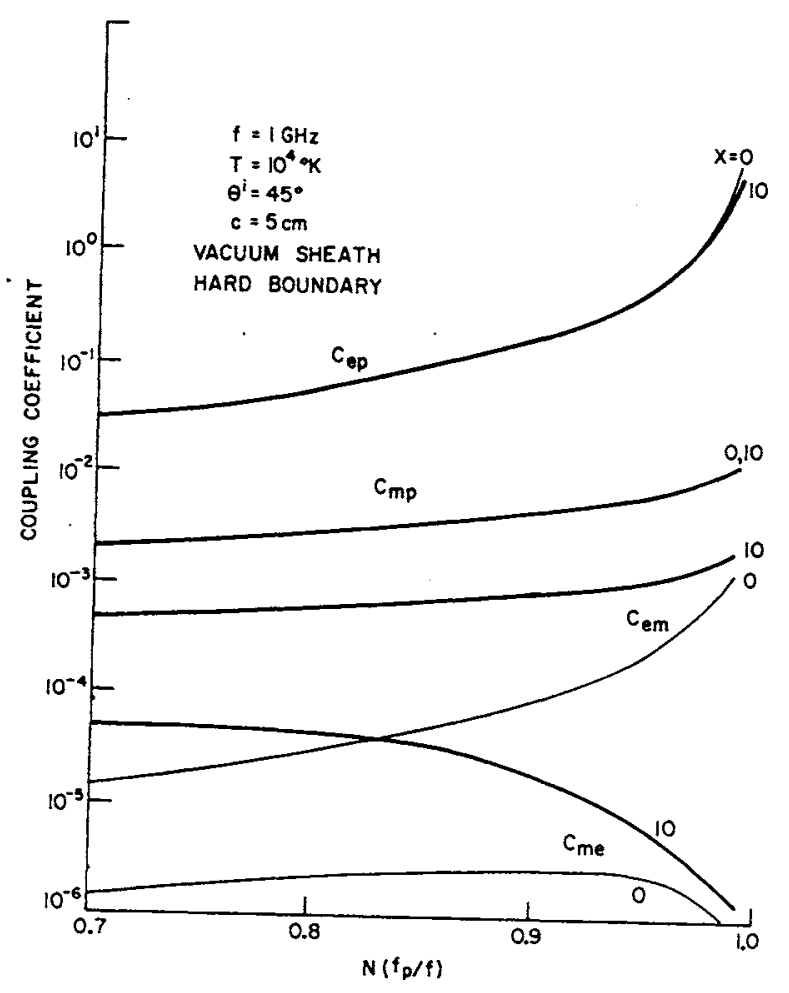

FIGURE 7. The coupling coefficients as a function of the plasma frequency to incident wave frequency ratio $\mathrm{N}$, for the vacuum-sheath model and hard boundary.

having the same values as the inhomogeneous-sheath results. Only for the EM-EK coupling coefficients do the results of the vacuum- and inhomogeneous-sheath models differ appreciably, the inhomogeneous-sheath results decreasing in magnitude while the vacuumsheath results remain constant, for increasing sheath thicknesses.

We can conclude from these results, as has been previously concluded by Miller and Olte (1966) in connection with the surface currents excited on a plasma immersed cylinder by EM and EK waves, that the vacuum sheath approximates the inhomogeneous sheath quite well in predicting the scattering properties of the plasma immersed cylinder, if the vacuum-sheath thickness is about 0.6 the corresponding inhomogeneous-sheath thickness. An exception to this is the finding noted above that the EM-EK coupling is exaggerated by the vacuum-sheath model as compared with the more realistic inhomogeneous sheath results.

We also conclude that while the sheath and plasma compressibility do lead to polarization conversion of the incident EM wave, the effect is small and has little infuence on the primary scattering coefficients. In addition, the cross-coupled field components are generally orders of magnitude less than the primary

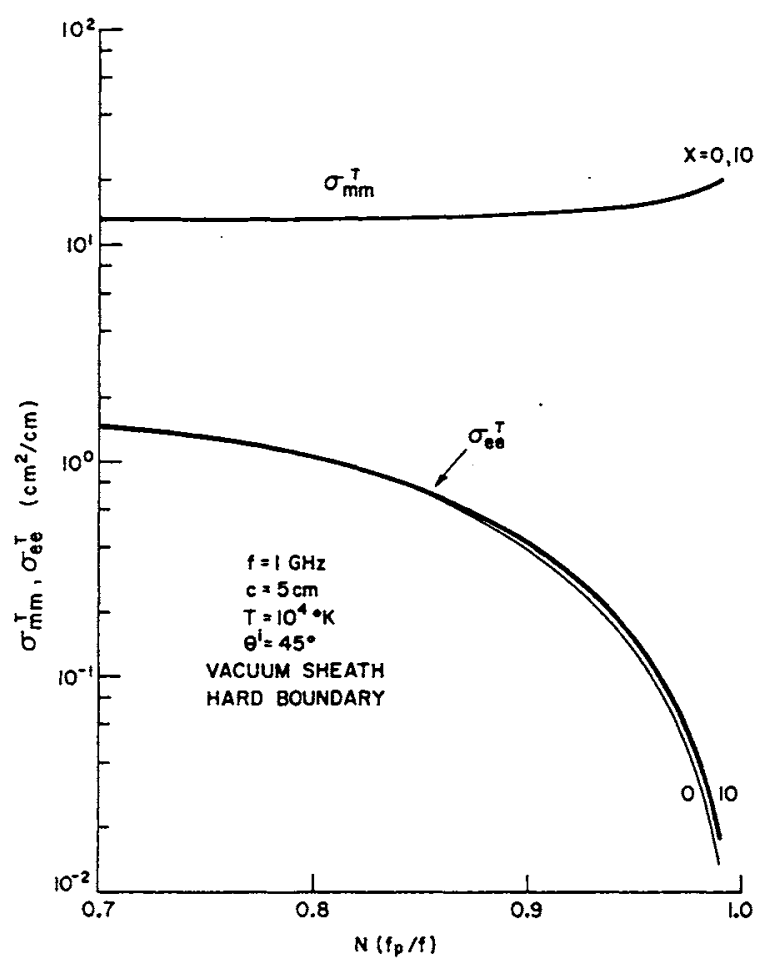

FIGURE 8. The primary cross sections as a function of $\mathrm{N}$ for the vacuum-sheath model and the hard boundary.

scattered fields, and hence would probably be difficult to observe experimentally.

\section{References}

Cohen, M. H. (1962), Radiation in a plasma, Pt. II, Equivalent Sources, Phys. Rev. 126, No. 2, 339-397.

Gabor, D., E. A. Ash, and D. Dracott (1955), Langmuir's paradox, Nature 176, No. 4489, 916-919.

Gierke, E., W. Ott, and F. Schwirzke (1961), Proc. Fifth Internatl. Conf. on Ionized Gases, Munich, 2, p. 1412.

Harp, R., and G. S. Kino, Experiments on the plasma sheath, Sixth Internatl. Conf. on Ionization Phenomena in Gases, Paris.

Miller, E. K. (1966), The excitation of surface currents on a plasma immersed cylinder by electromagnetic and electrokinetic waves, High Altitude Eng. Lab. Rept. 05627-4-S, Univ. of Mich., Ann Arbor, Mich.

Miller, E. K., and A. Olte (1966), Excitation of surface currents on a plasma immersed cylinder by electromagnetic and electrokinetic waves, Pt. II, the inhomogeneous sheath, Radio Sci. 1 (New Series), No. 12, 1425-1433.

Seshadri, S. R., I. L. Morris, and R. J. Mailloux (1964), Scattering by a perfectly conducting cylinder in a compressible plasma, Can. J. Phys. 42, No. 3, 465-476.

Wait, J. R. (1965), Scattering of electromagnetic and electroacoustic waves by a cylindrical object in a compressible plasma, Radio Sci. J. Res. NBS 69D, No. 2, 247-256.

(Paper 2-12-307) 